\title{
Light-absorption selection of nanoparticles and nanofluids containing nanoparticles for their effective heating by solar radiation
}

\author{
Victor K. Pustovalov ${ }^{1} \cdot$ Liudmila G. Astafyeva $^{2} \cdot$ Wolfgang Fritzsche $^{3}$
}

Received: 18 March 2017 / Accepted: 31 May 2017/Published online: 6 June 2017

(c) Springer International Publishing Switzerland 2017

\begin{abstract}
Light-absorption selection of single nanoparticle for solar radiation requires the simultaneous fulfillment of all of the following novel conditions-maximal close (overlap) of the dependence of nanoparticle efficiency absorption factor on wavelength with the dependence solar irradiance, predominant role of nanoparticle absorption over its scattering, the use of maximal values of nanoparticle efficiency absorption factor and its size. These results highlight the possibility for effective application of single homogeneous $\mathrm{Ti}$ and core-shell $\mathrm{Ti}-\mathrm{TiO}_{2}, \mathrm{Ni}-\mathrm{NiO}$ nanoparticles with radii of about $75 \mathrm{~nm}$ as perfect absorbers for solar radiation in the complete optical spectrum 250-2500 nm. Light-absorption conditions for nanofluids include dominant radiation absorption by presented nanoparticles with selected properties and concentrations of about $1 \times 10^{9}, 1 \times 10^{10} \mathrm{~cm}^{-3}$ under the solar absorption by water in the spectral interval of $250-1000 \mathrm{~nm}$, which includes $\sim 70 \%$ of whole solar energy. Presented results can be applied for applications in the development of novel working nanofluids for direct absorption solar collectors. These conditions can be used for the selection of various nanoparticles from different materials and structures for other optical radiation sources.
\end{abstract}

Victor K. Pustovalov

pustovalovv@mail.ru

1 Belarusian National Technical University, Nezavisimosti pr. 65, 220013 Minsk, Belarus

2 B.I. Stepanov Institute of Physics, National Academy of Sciences of Belarus, Nezavisimosti pr. 68, 220072 Minsk, Belarus

3 Leibniz Institute of Photonic Technology, A.-Einstein-Str. 9, 07745 Jena, Germany
Keywords Solar radiation · Light-absorption conditions · Nanoparticles $\cdot$ Nanofluids

\section{Introduction}

In recent years, the solar radiation absorption and heating of nanoparticles (NPs) and nanofluids containing NPs, including the steam nanobubbles formation, became an important area of photothermal solar energy conversion [1-12]. These phenomena are used in application of NPs and nanofluids in solar energy harvesting, radiation chemistry and catalysis, the use of steam in autoclaves, etc. Successful applications of NPs and nanofluids for solar energy trapping and conversion are based on their appropriate optical and other properties, among them first of all, the high radiation absorption by NPs because of plasmon resonance $[13,14]$. The attempts to search for the appropriate NPs from suitable materials for successful applications in solar energy harvesting are ongoing [15-17].

Metallic NPs are of special interest for photothermal (PT) and solar energy applications because of their prominent plasmonic and thermo-optical properties [1-17]. Also metal oxide core-shell NPs are very interesting due to their solar energy absorption. Some metal NPs undergo the action of intensive optical (solar) radiation, their heating and subsequent natural oxidation in gaseous or liquid media, containing of oxygen components (air, liquid, vapor) in the process of their applications, that leads to the formation of thin oxide shell with the thicknesses of about $5-10 \mathrm{~nm}$ on NPs. This fact should be taken into account for possible solar thermal applications of metallic NPs.

Solar thermal collectors are used for solar energy absorption and conversion in other forms of energy. They are heat exchangers that are used to absorb and 
transform solar radiation energy to thermal energy of the transport liquid. It is proposed to directly absorb the solar energy within the fluid volume and to sharply enhance the efficiency of collectors-the so-called direct absorption solar collector (DASC) [6-9]. DASCs have been used for a variety of applications such as water and air heating.

However, the efficiency of DASCs is found to be limited by the absorption properties of the working fluid, which is very poor for typical fluids (water) used in solar collectors. It is proposed to use NPs in fluids (nanofluids) as volumetric absorber in DASCs. Nanofluids are a mixture of base fluid and NPs. Nanofluids have intensified thermophysical properties, such as thermal conductivity, viscosity and convective heat transfer coefficients, compared with conventional fluids.

Because of the tremendous scientific and technological advances made during the last years, the ongoing research and development nanotechnology has an important function in solar thermal technology. Many types of NPs from different materials with various structures, sizes, shapes are investigated and produced now. NPs offer the potential of improving the radiation properties of liquids leading to an increase in the efficiency of DASCs. The main problem is the selection of suitable NPs to provide excellent optical properties of nanofluids. The results of this article can be used for the solution of this task. The selection of appropriate NPs and nanofluids for the improvement and manipulation of the NP plasmon resonances is very important for solar energy absorption and harvesting [1-19]. On the other side, a comparative analysis of optimal parameters of various metallic and metal-its oxide core-shell NPs for using them as PT agents in solar nanotechnology is still missing.

In the following, a complex and extensive investigation of the light-absorption conditions for spherical metallic and metal-its oxide core-shell NPs and nanofluids containing these NPs has been carried out for their interaction with solar radiation on the base of computer modeling.

\section{Materials and method}

On the base of the analysis of optical properties of different NPs [18, 19], metallic Ni, Ti and metal core and oxide shell $\mathrm{Ni}-\mathrm{NiO}, \mathrm{Ti}-\mathrm{TiO}_{2} \mathrm{NPs}$ with the radii of about $75 \mathrm{~nm}$ were chosen for the investigation. Efficiency factors of absorption $K_{\mathrm{abs}}$ and scattering $K_{\mathrm{sca}}$ of radiation [13] by mentioned NPs numerically calculated on the base of Mie theory [13].

\section{Results and discussion}

\section{Light-absorption conditions for a single nanoparticle}

The following parameters of NP, solar radiation and ambient medium are of special interest for the light-absorption conditions: solar radiation-

1. dependence of solar irradiance (radiation intensity) $I_{\mathrm{S}}$ on the wavelength $\lambda[20]$;

spherical nanoparticle-

1. type of NP material (metal) with its optical indexes of refraction $n_{0 \lambda}$ and absorption $\varkappa_{0 \lambda}$,

2. NP structure (homogeneous, core-shell, etc.),

3. NP radius $r_{0}$,

4. efficiency factors of absorption $K_{\text {abs }}$ and scattering $K_{\text {sca }}$ of radiation with wavelength $\lambda$ by NP with radius $r_{0}$;

surrounding medium-

1. coefficient of thermal conductivity $k_{\mathrm{m}}$,

2. optical index of refraction $n_{\lambda}$.

The achievement of an optimal combination of a maximal absorption and a minimal scattering of radiation by NP is very important for the efficiency of solar energy harvesting. The parameter $P_{1}=K_{\text {abs }} / K_{\text {sca }}$ is greater than 1 , $P_{1}>1\left(\right.$ or $\left.P_{1} \gg 1\right)$, and the factor of absorption $K_{\mathrm{abs}}$ is greater (or much greater in favorable cases) than the scattering factor $K_{\text {sca }}$ in the cases of predominant role of absorption. This situation allows to achieve maximal efficiency of solar radiation interaction with NP, resulting in maximal NP heating [18-22].

The achievement of maximal value $T_{0 \max }$ of NP temperature under $\mathrm{CW}$ solar irradiation is described by [18-22]

$\Delta T_{0}=T_{0 \max }-T_{\infty}=\frac{r_{0}}{4 k_{\mathrm{m}}} \int_{\lambda_{1}}^{\lambda_{2}} I_{S}(\lambda) K_{\mathrm{abs}}\left(r_{0}, \lambda\right) \mathrm{d} \lambda$

$T_{0}$ is the NP temperature, $T_{\infty}$ is the initial NP and ambient medium temperature, $k_{\mathrm{m}}$ is the coefficient of medium heat conduction, and the wavelengths $\lambda_{1}, \lambda_{2}$ are the boundaries of the optical spectrum under consideration. This equation has been obtained from NP energy conservation equation taken into account radiation absorption, NP heating and heat loss inside the surrounding medium because of heat conduction [18-22]. The coefficient of heat conduction has constant value for water $k_{\mathrm{m}}=6.10^{-3} \mathrm{~W} / \mathrm{cmK}$ [23], and this assumption is based on the rather small temperature interval of NP heating of smaller than $100{ }^{\circ} \mathrm{C}$. 
For fixed value of $k_{\mathrm{m}}$, the maximal value of $\Delta T_{0}$ is determined by the maximal value of $r_{0} \int_{\lambda_{1}}^{\lambda_{2}} I_{\mathrm{S}}(\lambda) K_{\mathrm{abs}}$ $\left(r_{0}, \lambda\right) \mathrm{d} \lambda$ and reached by the simultaneous use of maximal values of $r_{0}$ and the presented integral. A maximal value of NP heating (depending on integral) is achieved under maximal close (approach) between the dependencies of selected $K_{\mathrm{abs}}(\lambda)$ and the well-known dependence of solar irradiance $I_{\mathrm{S}}(\lambda)$. It is also necessary to use the selected NP with maximal possible values $r_{0}^{\max }$ and $K_{\mathrm{abs}}^{\max }$ [18-22]. The values of indexes $n_{0 \lambda}, x_{0 \lambda}$ and $n_{\lambda}$ [24, 25] were used for calculation of the factors of absorption $K_{\mathrm{abs}}$ and scattering $K_{\text {sca }}$.

Figure 1 presents the dependencies of solar irradiance $I_{\mathrm{S}}$, absorption efficiency factor $K_{\mathrm{abs}}$ and parameter $P_{1}$ for homogeneous $\mathrm{Ti}$ and Ni NPs with the radius $r_{0}=75 \mathrm{~nm}$ and core-shell $\mathrm{Ti}-\mathrm{TiO}_{2}$ as well as $\mathrm{Ni}-\mathrm{NiO}$ NPs with the outer radius $r_{1}=75 \mathrm{~nm}$ (core radius $r_{0}=65 \mathrm{~nm}$, the thickness of oxide shell is $\Delta r_{1}=10 \mathrm{~nm}, r_{1}=r_{0}+\Delta r_{1}$ ) on $\lambda$ in the spectral interval $\lambda_{1}=250 \mathrm{~nm}$ and $\lambda_{2}=2500 \mathrm{~nm}$ that contains approximately $\approx 99 \%$ of whole solar energy. It used optical indexes.

The dependencies of $K_{\mathrm{abs}}$ on $\lambda$ for Ti with $r_{0}=75 \mathrm{~nm}$ and $\mathrm{Ti}-\mathrm{TiO}_{2}$ NPs with $r_{1}=75 \mathrm{~nm}$ are very close to the dependence of $I_{\mathrm{S}}(\lambda)$ in the spectral interval of $\lambda \sim 500-$ $2500 \mathrm{~nm}$ with $K_{\mathrm{abs}}^{\mathrm{max}} \approx 1.76$ and $K_{\mathrm{abs}}^{\mathrm{max}} \approx 1.86$ accordingly. The parameter $P_{1}$ for Ti NPs with $r_{0}=75 \mathrm{~nm}$ is approximately equal 1 in the interval $250-700 \mathrm{~nm}$. $P_{1}$ is bigger than 1 and achieves the values of $P_{1} \sim 10-20$ in the spectral interval $700-2500 \mathrm{~nm}$. The maximum of a
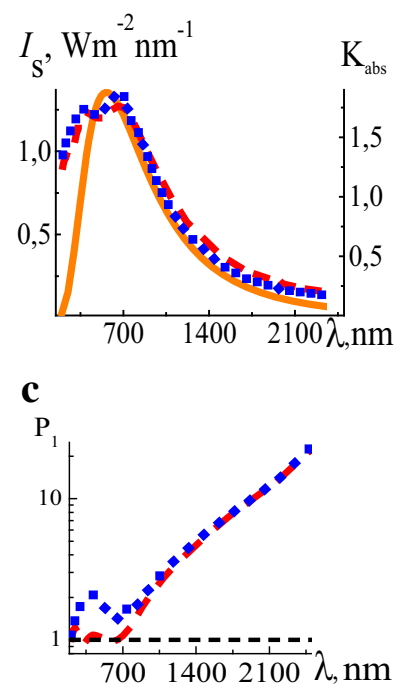

b

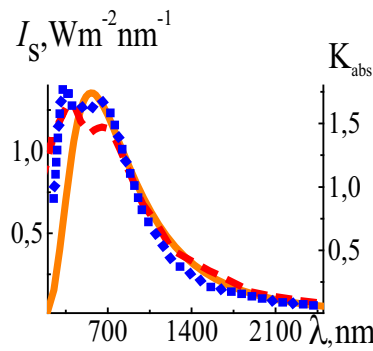

d

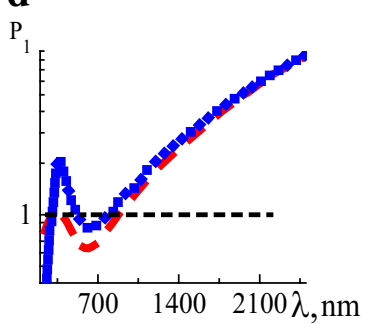

Fig. 1 The dependencies of solar irradiance $\mathrm{I}_{\mathrm{S}}(\mathbf{a}, \mathbf{b}$, solid, orange $)$, absorption efficiency factor $K_{\text {abs }}(\mathbf{a}, \mathbf{b})$ and parameter $P_{1}(\mathbf{c}, \mathbf{d})$ for Ti $(\mathbf{a}$, c), $\mathrm{Ni}(\mathbf{b}, \mathbf{d}) \mathrm{NPs}$ (dashed lines, red) and for $\mathrm{Ti}^{-\mathrm{TiO}_{2}}(\mathbf{a}, \mathbf{c}), \mathrm{Ni}-\mathrm{NiO}(\mathbf{b}$, d) NPs (dotted lines, blue) with the radii $r_{0}, r_{1}=75 \mathrm{~nm}$ on $\lambda$. Horizontal dashed lines (c, d) denote the value of $P_{1}=1$ (color figure online) parameter $P_{1}$ has been created for $\mathrm{Ti}-\mathrm{TiO}_{2}$ NPs with $r_{1}=75 \mathrm{~nm}$ at $\lambda \sim 400 \mathrm{~nm}$. The value of $P_{1}$ is equal to $P_{1} \sim 1.5-2$ in the spectral interval $250-800 \mathrm{~nm}$, and it is sharply increased up to $P_{1} \sim 10-20$ with increase in $\lambda$ in the spectral interval $800-2500 \mathrm{~nm}$.

The dependencies of $K_{\mathrm{abs}}$ on $\lambda$ for Ni and Ni-NiO NPs with $r_{0}, r_{1}=75 \mathrm{~nm}$ are ideally close (practically overlaps) to the dependence of $I_{\mathrm{S}}(\lambda)$ in the spectral interval of $\lambda \sim 500-2500 \mathrm{~nm}$ with $K_{\mathrm{abs}}^{\mathrm{max}} \approx 1.55$ and $K_{\mathrm{abs}}^{\max } \approx 1.65$ accordingly. The parameter $P_{1}$ is smaller than $1, P_{1}<1$, for $\mathrm{Ni}$, NiO NPs with $r_{0}, r_{1}=75 \mathrm{~nm}$ in the important spectral intervals $250-850 \mathrm{~nm}$ and $550-750 \mathrm{~nm}$ accordingly from the point of view on amount of solar energy placed there. The parameter $P_{1}$ is bigger than $1, P_{1}>1$, for Ni NPs with $r_{0}=75 \mathrm{~nm}$ in the spectral interval $\lambda \approx 850-2500 \mathrm{~nm}$, and it achieves the values of $P_{1} \sim 10$ with increasing $\lambda$ in infrared region. The value of $P_{1}$ for $\mathrm{NiO}$ NPs is bigger than 1 in the intervals 300-500, $800-2500 \mathrm{~nm}$, and it has the maxima of $P_{1} \sim 2$ at $\lambda \sim 400 \mathrm{~nm}$ and $P_{1} \sim 10$ at $\lambda \sim 2500 \mathrm{~nm}$.

The presence of an oxide shell leads to an increase in absorption compared to scattering of radiation by twolayered NPs in the important spectral intervals 250-800 nm for $\mathrm{Ti}_{-}-\mathrm{TiO}_{2}$ NPs and in 300-700 nm for Ni$\mathrm{NiO}$ NPs in comparison with pure $\mathrm{Ti}$ or $\mathrm{Ni} \mathrm{NPs}$ and improves the possibility of two-layered NPs applications with enhanced performance for energy absorption.

These results highlight the possibility for effective application of $\mathrm{Ti}, \mathrm{Ti}-\mathrm{TiO}_{2}$ and $\mathrm{Ni}-\mathrm{NiO}$ NPs and to a lower degree for Ni NPs with $r_{0}, r_{1}=75 \mathrm{~nm}$ as perfect absorbers for solar radiation in the complete optical spectrum 250-2500 nm.

For effective absorption of solar radiation by single NP and the realization of maximal value of the NP heating $\Delta T_{0}$ by solar radiation, it is necessary to select NP materials (metals) with their parameters and NP structure (homogeneous, layered, etc.). Therefore, it needs simultaneous fulfillment of all of the following conditions:

1. The dependence of NP $K_{\mathrm{abs}}$ on $\lambda$ should be maximally close (overlap) to the dependence $I_{\mathrm{S}}(\lambda)$;

2. The fulfillment of the condition $K_{\mathrm{abs}}>K_{\mathrm{sca}}, P_{1}>1$;

3. The use of maximal value of $K_{\mathrm{abs}}^{\max }\left(r_{0}^{\max }, \lambda\right)$;

4. The use of maximal value $r_{0}^{\max }$ that allows to fulfill the previous conditions.

\section{Light-absorption conditions of solar radiation for nanofluids}

Three different light-absorption scenarios can be realized for nanofluids containing NPs in an surrounding liquid under optical (solar) radiation action: 
1. rapid heating of NPs above $100{ }^{\circ} \mathrm{C}$ with vapor production near NPs without significant heat exchange with surrounding cold liquid (water) [10-12],

2. heating of NPs and water simultaneously and lower than $100{ }^{\circ} \mathrm{C}$ for thermal solar energy applications [1-9],

3. heating only of the liquid (water) and NPs remain cold.

The third situation looks like as exotic one but is included for the sake of completeness.

It is interesting to determine the contribution of water and NPs separately in absorption and scattering of solar radiation by nanofluids in the complete spectral interval of 250$2500 \mathrm{~nm}$. Figures 2 and 3 present the dependencies of the coefficients of scattering $\alpha_{\mathrm{sca}}^{W}$ and absorption $\alpha_{\mathrm{abs}}^{W}$ of radiation by water [25] and the calculated coefficients of scattering $\alpha_{\mathrm{sca}}^{N}$ and absorption $\alpha_{\mathrm{abs}}^{N}$ radiation by NP ensembles from Ti and $\mathrm{Ni}$ NPs with radii $r_{0}=75 \mathrm{~nm}, \mathrm{Ti}-\mathrm{TiO}_{2}$ and $\mathrm{Ni}-\mathrm{NiO} \mathrm{NPs}$ with radii $r_{1}=75 \mathrm{~nm}$ and with $\mathrm{NP}$ concentrations $N_{0}=1 \times 10^{9}, 1 \times 10^{10} \mathrm{~cm}^{-3}$, and the dependence of solar radiation intensity $I_{\mathrm{S}}$ on $\lambda$. The radiation absorption and scattering by NP ensemble with higher concentration $\left(N_{0}=1 \times 10^{10} \mathrm{~cm}^{-3}\right)$ are bigger than for the lower concentrated $\left(N_{0}=1 \times 10^{9} \mathrm{~cm}^{-3}\right)$ solution.

In the spectral interval $250-1000 \mathrm{~nm}$, solar radiation absorption by water is much smaller than radiation absorption by NPs with concentration $N_{0}=1 \times 10^{9}$, $1 \times 10^{10} \mathrm{~cm}^{-3}$. As a result, solar radiation absorption in the spectral interval $250-1000 \mathrm{~nm}$ is determined by and is dominated by the influence of NPs. However, in the spectral interval $\lambda>1250 \mathrm{~nm}$, water is the dominating factor (up to fivefold and higher) in radiation absorption for $N_{0}=1 \times 10^{9}, 1 \times 10^{10} \mathrm{~cm}^{-3}$. The spectral interval $\lambda \sim 1000-1250 \mathrm{~nm}$ is a transition zone from the dominating influence of NPs to the one of water on solar radiation absorption. Approximately $\sim 20 \%$ of whole solar radiation energy concentrates in the spectral interval $1250<\lambda<2500 \mathrm{~nm}$ (see Figs. 2, 3). In this spectral range, the radiation absorption coefficient for water is $\alpha_{\mathrm{abs}}^{W}$ $\sim 10^{1}-10^{2} \mathrm{~cm}^{-1}$ [24]. Therefore, absorption of solar radiation in this spectral interval will be realized in water thin layer with the thickness of about $\sim 10^{-1}-10^{-2} \mathrm{~cm}$. Coefficient $\alpha_{\mathrm{abs}}^{N}$ is approximately equal $\alpha_{\mathrm{abs}}^{N} \approx 2 \times 10^{-1}$ $\mathrm{cm}^{-1} \quad$ (for $\quad N_{0}=1 \times 10^{9} \mathrm{~cm}^{-3}$ ) and $\approx 2 \mathrm{~cm}^{-1}$ $\left(N_{0}=1 \times 10^{10} \mathrm{~cm}^{-3}\right)$, and the thickness of a nanofluid layer with intensive radiation absorption by NP will be approximately equal $5-0.5 \mathrm{~cm}$ in the spectral interval 250-1300 nm. These results allow to estimate the characteristics of nanofluids with selected values of $r_{0}, N_{0}$, types of NP metal and the thickness of absorbing nanofluid layer.

Scattering by $\mathrm{Ti}, \mathrm{T}+\mathrm{TiO} 2$ and $\mathrm{NiO} \mathrm{NPs}$ is approximately equal their optical absorption in the spectral interval 250-800 $\mathrm{nm}$. The scattering decreases in comparison with absorption with increase in $\lambda$. Radiation scattering by water is significant only in the UV spectral region (see Fig. 2) and is approximately equal zero for $\lambda>500 \mathrm{~nm}$.

The difference between the coefficients of absorption $\alpha_{\mathrm{abs}}^{N}$ and scattering $\alpha_{\mathrm{sca}}^{N}$ of solar radiation by NP ensemble with $N_{0}=1 \times 10^{10} \mathrm{~cm}^{-3}$ and the coefficient of absorption $\alpha_{\text {abs }}^{W}$ of radiation by ambient liquid (water) should provide the fulfillment of the next conditions:

1. $\alpha_{\mathrm{abs}}^{N}=\pi N_{0} r_{0}^{2} K_{\mathrm{abs}}>\alpha_{\mathrm{abs}}^{W}, \alpha_{\mathrm{abs}}^{N} \sim \alpha_{\mathrm{sca}}^{N}, K_{\mathrm{abs}} \sim K_{\mathrm{sca}}$. $1000>\lambda>250 \mathrm{~nm}$

2. $\alpha_{\mathrm{abs}}^{N}=\pi N_{0} r_{0}^{2} K_{\mathrm{abs}} \sim \alpha_{\mathrm{abs}}^{W}, \alpha_{\mathrm{abs}}^{N}>\alpha_{\mathrm{sca}}^{N}, K_{\mathrm{abs}}>K_{\mathrm{sca}}$. $1250>\lambda>1000 \mathrm{~nm}$

3. $\alpha_{\mathrm{abs}}^{N}=\pi N_{0} r_{0} K_{\mathrm{abs}}<\alpha_{\mathrm{abs}}^{W}, \alpha_{\mathrm{abs}}^{N}>\alpha_{\mathrm{sca}}^{N}, K_{\mathrm{abs}}>K_{\mathrm{sca}}$. $2500>\lambda>1250 \mathrm{~nm}$

The optical properties of nanofluids are determined by the summarized action of optical properties of water (liquid) and NPs ensemble. Light-absorption conditions a

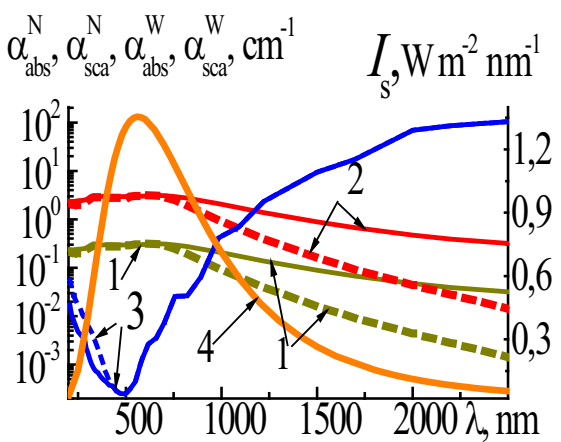

Fig. 2 Dependencies of the coefficients of scattering $\alpha_{\mathrm{sca}}^{N}$ (dashed) and absorption $\alpha_{\mathrm{abs}}^{N}$ (solid) of radiation by NP ensembles from Ti (a) NPs with radii $r_{0}=75 \mathrm{~nm}$ and $\mathrm{Ti}-\mathrm{TiO}_{2}$ (b) NPs with radii $r_{1}=75 \mathrm{~nm}$ and with $\mathrm{NP}$ concentrations $N_{0}=1 \times 10^{9} \quad(1)$, b

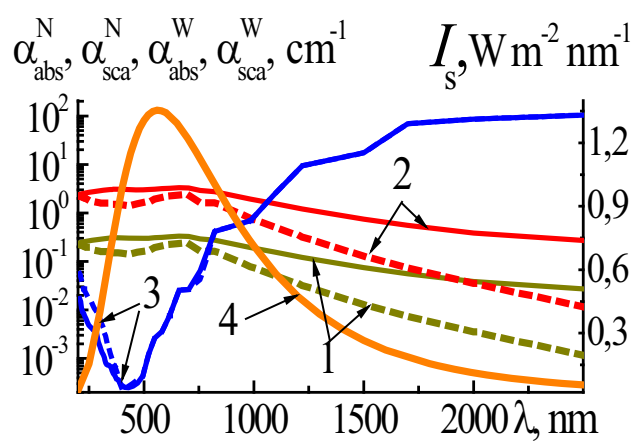

$1 \times 10^{10}(2) \mathrm{cm}^{-3}$, the coefficients of scattering $\alpha_{\mathrm{sca}}^{W}$ (dashed, blue) and absorption $\alpha_{\mathrm{abs}}^{W}$ (solid, blue) of radiation by water (3) and the dependence of solar radiation intensity $I_{\mathrm{S}}$ on $\lambda(4$, orange) (color figure online) 
$\mathbf{a}$

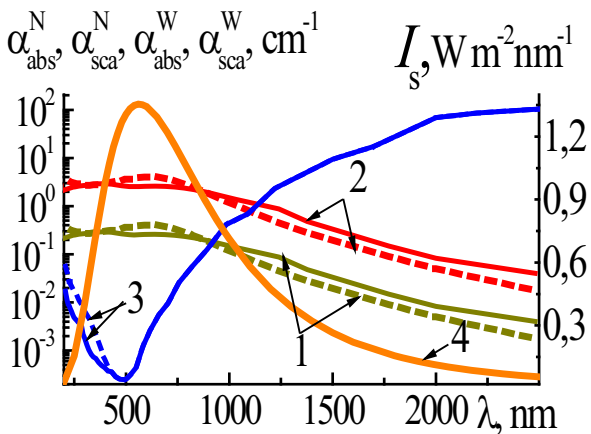

Fig. 3 Dependencies of the coefficients of scattering $\alpha_{\mathrm{sca}}^{N}$ (dashed) and absorption $\alpha_{\text {abs }}^{N}$ (solid) of radiation by NP ensembles from Ni (a) NPs with radii $r_{0}=75 \mathrm{~nm}$ and $\mathrm{Ni}-\mathrm{NiO}$ (b) NPs with radii $r_{1}=75 \mathrm{~nm}$ and with $\mathrm{NP}$ concentrations $N_{0}=1 \times 10^{9} \quad(1)$,

for nanofluids include dominant radiation absorption by nanoparticles with selected properties and concentrations compared to the solar absorption by water in the spectral interval $250-1000 \mathrm{~nm}$, which includes $\sim 70 \%$ of whole solar energy. An analysis of optical properties of NPs and nanofluids, concentrations and radii of NPs can give us the appropriate NP types for the realization of effective absorption of optical (solar) radiation by NPs and nanofluid for photothermal solar energy conversion applications.

\section{Environmental applications of the results}

Sustainable energy generation is one of the most important challenges of our society today. Energy resources based on fossil fuels are still dominating over the past decades with the highest share in global energy consumption. For example, electricity consumption is increasing year by year and electricity generation uses fossil fuels such as coal, petroleum and natural gas. On the other hand, the burning of fossil fuels results in unwillingness environmental consequences, such as air pollution, acid rain, ozone layer depletion and global warming climate change.

The protection of the global environment requires clean energy sources, because the generation of clean energy is crucial due to the growing significance of environmental problems. The renewable and clean energy technologies can meet much of the growing energy demand without harming the environment. Solar power is the most environmentally compatible permanent source and a key item in renewable energy technologies because it provides a nearly unlimited, clean and environmentally friendly energy. Therefore, a method of efficient solar energy harvesting and conversion must be developed and used as an alternative in the most vulnerable applications of fossil fuels. b

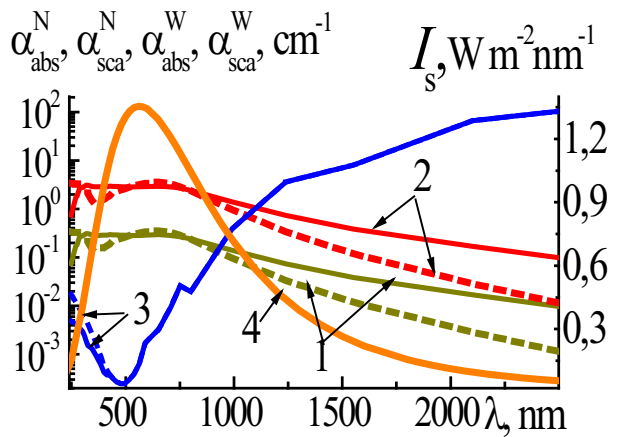

$1 \times 10^{10}(2) \mathrm{cm}^{-3}$, the coefficients of scattering $\alpha_{\mathrm{sca}}^{W}$ (dashed, blue) and absorption $\alpha_{\mathrm{abs}}^{W}$ (solid, blue) of radiation by water (3) and the dependence of solar radiation intensity $I_{\mathrm{S}}$ on $\lambda$ (4, orange) (color figure online)

Solar energy will be sustainably utilized in the near future instead of other alternative energy forms owing to its unlimited availability and desirable environmental and safety aspects. Solar energy provides an increasing share of the world's total energy demand and is growing faster than liquid fuels, natural gas and coal.

DASCs are now one of the most used thermal solar energy collectors with minimal harmful influence on environment. In general, the increase in DASC efficiency can be used for improvement of solar thermal energy consumption and for the decrease in pollution influence of conventional energy production technologies on environment.

\section{Conclusions}

Novel light-absorption conditions have been firstly formulated for single NP and for nanofluids, containing NPs that can be used for effective absorption of solar radiation. Light-absorption selection of single NP for solar radiation requires the simultaneous fulfillment of all of the following conditions - maximal close (overlap) of the dependence of nanoparticle efficiency absorption factor on wavelength with the same dependence solar irradiance, predominant role of nanoparticle absorption over its scattering, the use of maximal values of nanoparticle efficiency absorption factor and its size. These results highlight the possibility for effective application of single homogeneous $\mathrm{Ti}$ and core-shell $\mathrm{Ti}-\mathrm{TiO}_{2}, \mathrm{Ni}-\mathrm{NiO}$ nanoparticles with radii of about $75 \mathrm{~nm}$ as perfect absorbers for solar radiation in the complete optical spectrum 250-2500 nm.

Light-absorption conditions for nanofluids include dominant radiation absorption by presented NPs with selected properties and concentrations of about $1 \times 10^{9}$, $1 \times 10^{10} \mathrm{~cm}^{-3}$ under the solar absorption by water in the 
spectral important interval of 250-1000 nm, which includes $\sim 70 \%$ of whole solar energy. Dominant absorption by water is achieved in the spectral interval for wavelength greater than $1250 \mathrm{~nm}$.

The increase in NP concentrations over the presented values does not increase the NPs absorption of solar radiation compared to water absorption. In the spectral interval $1250<\lambda<2500 \mathrm{~nm}$, the radiation absorption coefficient for water is $\alpha_{\mathrm{abs}}^{W} \sim 10^{1}-10^{2} \mathrm{~cm}^{-1}$ [25] and, therefore, absorption of solar radiation will be realized in water thin layer with the thickness of about $\sim 10^{-1}-10^{-2} \mathrm{~cm}$. On the other hand, real thickness of absorption solar collectors is no more than $3-5 \mathrm{~cm}$.

These conditions can be used for the selection of various nanoparticles and nanofluids from different materials and structures for other optical radiation sources. Selection of suitable nanoparticles, with the parameters, that fulfill the formulated conditions, includes the choice of their structure (homogeneous, core-shell, etc.), material (metal, oxide, etc.) of core, shell or homogeneous NPs, size (their radii, thicknesses of oxide shells), optical indexes of absorption and refraction of NP material and surrounding medium. The selection of novel nanofluids includes the choice of suitable NPs (type of NP materials, NP radii, their concentrations) and fluid (optical, thermophysical, etc.) parameters for effective absorption of optical (solar) radiation.

This paper contains novel information about the lightabsorption conditions for the selection of nanoparticles and nanofluids under solar radiation with concrete recommendations for their types and parameters that allow to use of selected NPs and nanofluids with determined parameters for the nanofluid-based direct absorption solar collectors (DASCs). Nanoparticles with the selected characteristics can realize their effective heating by solar radiation simultaneously and lower than $100{ }^{\circ} \mathrm{C}$ for thermal solar energy applications.

Presented results can be applied for applications in the development of novel working nanofluids for DASCs. The fulfillment of formulated conditions can be used for sharp increase in efficiency of solar absorption by nanofluids and applications of novel results for DASCs.

\section{References}

1. Tang Y, Vlahovic B (2013) Metallic nano-particles for trapping light. Nanoscale Res Lett 8:65-72

2. Zhang H, Chen H-J, Du X, Wen D (2014) Photothermal conversion characteristics of gold nanoparticle dispersions. Sol Energy 100:141-147
3. Radousky HB, Liang H (2012) Energy harvesting: an integrated view of materials, devices and applications. Nanotechnology 23:502501

4. Mlinar V (2013) Engineered nanomaterials for solar energy conversation. Nanotechnology 24:042501

5. Hashemi SMH, Choi J-W, Psaltis D (2014) Solar thermal harvesting for enhanced photocatalytic reactions. Phys Chem Chem Phys 16:5137-5141

6. He Q, Wang S, Zeng S, Zheng Z (2013) Experimental investigations on photothermal properties of nanofluids for direct absorption solar thermal energy systems. Energy Convers Manage 73:150-157

7. Otanicar TP, Phelan PE, Golden JS (2009) Optical properties of liquids for direct absorption solar thermal energy systems. Sol Energy 83:969-977

8. Hossain M, Saidur R, Sabri M, Said Z, Hassani S (2015) Spotlight on available optical properties and models of nanofluids: a review. Renew Sustain Energy Rev 43:750-762

9. Gorji TB, Ranjbar AA (2017) A review on optical properties and application of nanofluids in direct absorption solar collectors (DASCs). Renew Sustain Energy Rev 72:10-32

10. Neumann O, Urban AS, Day J, Lal S, Halas NJ (2012) Solar vapor generation enabled by nanoparticles. ACS Nano 7:42-49

11. Fang Z, Zhen Y-R, Neumann O, Polman A, Garcia de Abajo FJ, Nordlander P, Halas NJ (2013) Evolution of light-induced vapor generation at a liquid-immersed metallic nanoparticle. Nano Lett 13:1736-1742

12. Polman A (2013) Solar steam nanobubbles. ACS Nano 7:15-18

13. Bohren CF, Huffman DR (1983) Absorption and scattering of light by small particles. Wiley, New York

14. Kreibig U, Vollmer M (1995) Optical properties of metal clusters. Springer, Heidelberg

15. Bardhan R, Grady NK, Ali T, Halas NJ (2010) Metallic nanoshells with semiconductor cores: optical characteristics modified by core medium properties. ACS Nano 4:6169-6179

16. Sonnefraud Y, Koh A, McComb D, Maier S (2012) Nanoplasmonics: engineering and observation of localized plasmon modes. Laser Photonics Rev 6:277-295

17. Grigoriev V, Bonod N, Wenger J, Stout B (2015) Optimizing nanoparticle designs for ideal absorption of light. ACS Photonics 2:263-270

18. Pustovalov VK, Astafyeva LG, Fritzsche W (2015) Analysis of optical properties of spherical metallic nanoparticles for effective absorption of solar radiation and their heating. Sol Energy 122:1334-1341

19. Pustovalov VK (2016) Thermo-optical properties of spherical homogeneous and core-shell nanoparticles and their applications. In: Aliofkhazraei M (ed) Handbook of nanoparticles. Springer, Berlin, pp 819-841

20. ASTM G-173-03-International standard ISO 9845-1 (1992)

21. Pustovalov VK (2011) Modeling of the processes of lasernanoparticle interaction taking into account temperature dependences of parameters. Laser Phys 21:906-912

22. Pustovalov VK (2016) Light-to-heat conversion and heating of single nanoparticles, their assemblies, and surrounding medium under laser pulses: review. RSC Adv 6:81266-81289

23. Kreith F, Black WZ (1980) Basic heat transfer. Harper and Row, New York

24. Refractive index database (2015). http://refractiveindex.info/

25. Hale G, Querry M (1973) Optical constants of water in the 250-nm to 250- $\mu \mathrm{m}$ wavelength region. Appl Opt 12:555-562 